ORIGINAL ARTICLE

\title{
Dyskinesias and associated psychiatric disorders following streptococcal infections
}

\author{
R C Dale, I Heyman, R A H Surtees, A J Church, G Giovannoni, R Goodman, B G R Neville
}

Arch Dis Child 2004;89:604-610. doi: 10.1136/adc.2003.031856

See end of article for authors' affiliations

.....................

Correspondence to: Dr R Dale, Neurosciences Unit, Wolfson Centre Institute of Child Health, Mecklenburgh Square, London WCIN 2AP, UK; R.Dale@ion.ucl.ac.uk

Accepted 19 August 2003

\begin{abstract}
Background: The classical extrapyramidal movement disorder following $\beta$ haemolytic streptococcus (BHS) infection is Sydenham's chorea (SC). Recently, other post-streptococcal movement disorders have been described, including motor tics and dystonia. Associated emotional and behavioural alteration is characteristic.

Aims: To describe experience of post-streptococcal dyskinesias and associated co-morbid psychiatric features presenting to a tertiary referral centre 1999-2002.

Methods: In all patients, dyskinetic movement disorders followed BHS pharyngeal infection. BHS infection was defined by pharyngeal culture of the organism, or paired streptococcal serology. Movement disorders were classified according to international criteria, and validated by experienced child neurologists. Psychiatric complications were defined using ICD-10 criteria using a validated psychiatric interview. Results: In the 40 patients, the following dyskinetic movement disorders were present: chorea $(n=20)$, motor tics $(n=16)$, dystonia $(n=5)$, tremor $(n=3)$, stereotypies $(n=2)$, opsoclonus $(n=2)$, and myoclonus $(n=1)$. Sixty five per cent of the chorea patients were female, whereas $69 \%$ of the tic patients were male. ICD-10 psychiatric diagnoses were made in $62.5 \%$. Using the same psychiatric instrument, only $8.9 \%$ of UK children would be expected to have an ICD-10 psychiatric diagnosis. Emotional disorders occurred in $47.5 \%$, including obsessive-compulsive disorder (27.5\%), generalised anxiety (25\%), and depressive episode (17.5\%). Additional psychiatric morbidity included conduct disorders (27.5\%) and hyperkinetic disorders (15\%). Psychiatric, movement, and post-streptococcal autoimmune disorders were commonly observed in family members. At a mean follow up of 2.7 years, $72.5 \%$ had continuing movement and psychiatric disorders.

Conclusion: Post-streptococcal dyskinesias occur with significant and disabling psychiatric co-morbidity and are potential autoimmune models of common "idiopathic" movement and psychiatric disorders in children. Multiple factors may be involved in disease expression including genetic predisposition, developmental status, and the patient's sex.
\end{abstract}

Abbreviations: $A D H D$, attention deficit-hyperactivity disorder; $B H S, \beta$ haemolytic streptococcus; OCD, obsessive-compulsive disorder;

PANDAS, paediatric autoimmune neuropsychiatric disorders associated with streptococcal infections; SC, Sydenham's chorea 


\begin{tabular}{|c|c|c|c|c|c|}
\hline \multirow{2}{*}{$\begin{array}{l}\text { Age of movement } \\
\text { disorder onset }(y) \text {, } \\
\text { sex }\end{array}$} & \multirow[b]{2}{*}{ Movement disorder } & \multicolumn{3}{|l|}{ ICD-10 psychiatric disorders } & \multirow{2}{*}{$\begin{array}{l}\text { Movement disorder course } \\
\text { (disease duration in years) }\end{array}$} \\
\hline & & Emotional & Conduct & Hyperkinetic & \\
\hline $1.2, \mathrm{M}$ & Dystonia, chorea & - & - & - & Relapsing remitting (1.8) \\
\hline $1.3, \mathrm{~F}$ & Chorea & - & - & - & Persistent (1) \\
\hline $1.5, \mathrm{M}$ & Chorea & $O C D$ & - & Hyperkinesis (oth.) & Persistent (13) \\
\hline $2, M$ & Tics & OCD, social phobia, depression & Conduct (other) & Hyperkinesis & Persistent (13) \\
\hline $2, \mathrm{~F}$ & Chorea, dystonia & - & - & - & Resolution (0.25) \\
\hline $2, M$ & Myoclonus, dystonia & Panic attacks, gen. anxiety & Conduct (other) & - & Relapsing remitting (5) \\
\hline $3, M$ & Tics & - & - & - & Relapsing remitting (4) \\
\hline $3, M$ & Tics & Sep. anxiety, gen. anxiety, OCD & ODD & - & Relapsing remitting (5) \\
\hline $3, \mathrm{~F}$ & Stereotypies & $O C D$ & ODD & - & Persistent (7) \\
\hline $4, M$ & Dystonia & - & - & - & Resolution (0.2) \\
\hline $4, F$ & Chorea & - & - & - & Persistent (0.5) \\
\hline $5, M$ & Tics & Sep. anxiety, gen. anxiety, OCD & - & Hyperkinesis & Persistent (5) \\
\hline $5, \mathrm{~F}$ & Tics & Trichotillomania, depression & Conduct (other) & - & Persistent (2) \\
\hline $6, M$ & Tics & OCD & - & - & Persistent (1) \\
\hline $6, M$ & Tics & - & - & - & Relapsing remitting (3) \\
\hline $7, \mathrm{~F}$ & Tics & OCD & - & - & Persistent (8) \\
\hline $7, M$ & Chorea, tremor & - & - & - & Relapsing remitting (7) \\
\hline $7, \mathrm{~F}$ & Tics, stereotypies & $O C D$ & - & - & Persistent (2) \\
\hline $7, \mathrm{~F}$ & Chorea & - & - & - & Persistent (0.5) \\
\hline $7, M$ & Tics & - & - & Hyperkinesis & Relapsing remitting (2) \\
\hline $7, M$ & Chorea & - & - & - & Persistent (2) \\
\hline $8, F$ & Chorea & - & ODD & - & Relapsing remitting (1.5) \\
\hline $8, \mathrm{~F}$ & Chorea & Gen. anxiety & - & Hyperkinesis & Resolution $(0.25)$ \\
\hline $8, M$ & Tics & - & - & - & Relapsing remitting (2) \\
\hline $8, M$ & Tics & - & Conduct (other) & - & Relapsing remitting (7) \\
\hline $8, M$ & Tics & $O C D$ & - & - & Persistent (4) \\
\hline $8, F$ & Tics & - & - & - & Relapsing remitting (1) \\
\hline $8, M$ & Dystonia, chorea & $\begin{array}{l}\text { Gen. anxiety, spec. phobia, } \\
\text { depression }\end{array}$ & - & - & Relapsing remitting (1.5) \\
\hline $10, M$ & Chorea & Gen. anxiety & ODD & Hyperkinesis & Persistent $(0.25)$ \\
\hline $10, F$ & Chorea & $\begin{array}{l}\text { Sep. anxiety, gen. anxiety, } O C D \text {, } \\
\text { social phobia, depression }\end{array}$ & ODD & - & Relapsing remitting (1.5) \\
\hline $10, \mathrm{~F}$ & $\begin{array}{l}\text { Chorea, tremor, } \\
\text { opsoclonus }\end{array}$ & Gen. anxiety & - & - & Resolution (0.2) \\
\hline $11, M$ & Chorea & - & - & - & Resolution (0.8) \\
\hline $11, \mathrm{~F}$ & Chorea & - & - & - & Resolution (0.35) \\
\hline $11, \mathrm{~F}$ & Chorea & Depression & - & - & Resolution (0.8) \\
\hline $12, \mathrm{~F}$ & Chorea & - & Conduct (other) & - & Resolution (0.25) \\
\hline $12, F$ & Tics & - & - & - & Resolution (0.2) \\
\hline $13, M$ & Tics & $\begin{array}{l}\text { Gen. anxiety, specific phobia, } \\
\text { OCD, depression }\end{array}$ & - & - & Relapsing remitting (2.5) \\
\hline $13, \mathrm{~F}$ & Chorea & $\begin{array}{l}\text { Gen. anxiety, specific phobia, } \\
\text { depression }\end{array}$ & ODD & - & Resolution (0.8) \\
\hline $14, M$ & Chorea & - & - & - & Resolution (0.25) \\
\hline $16, \mathrm{~F}$ & Tremor, opsoclonus & - & - & - & Persistent (0.8) \\
\hline
\end{tabular}

first century. In addition, we aimed to define the accompanying psychiatric disorders that are often more impairing than the extra movements. These conditions represent a putative autoimmune model of acquired movement and psychiatric disorders, which may provide insight into the neuroanatomy and neurobiology of common neuropsychiatric syndromes.

\section{METHODS}

\section{Patients}

Patients were referred between 1999 and 2002 by their general practitioner or paediatrician for investigation and management of an acute onset or relapsing movement disorder. All patients included in this report had disease onset shortly after streptococcal pharyngeal infection, or suffered two or more relapses after streptococcal pharyngeal infections. ${ }^{5}$ Evidence of $\beta$ haemolytic streptococcal (BHS) infection was evident in all patients, and was diagnosed when a clinical episode of pharyngitis occurred with laboratory evidence of BHS infection (growth of BHS organism on pharyngeal swab, or increased acute streptococcal serology with reduction in serology 3-6 months later).
Movement disorders were initially diagnosed by RCD, and conform to accepted diagnostic definitions. ${ }^{15}$ Movement disorders were video-recorded and reviewed by two experienced child neurologists (BGRN and RAHS) in order to validate the movement disorder classifications, with discussion and agreement on problematic disorders. Investigations for an alternative cause of chorea, dystonia, and myoclonus (copper, caeruloplasmin, antinuclear antibody, thyroid function tests, lactate, plasma amino acids, and urine organic acids) were normal in all patients. MRI brain scanning was performed in 26 and was normal in 23. Three patients had localised inflammatory changes in the basal ganglia seen on $\mathrm{T} 2$ weighted imaging.

Presence of psychopathology was assessed using the Strengths and Difficulties Questionnaire (SDQ), ${ }^{16}$ and the Development and Well-Being Assessment (DAWBA) ${ }^{17}$ by RCD. The DAWBA is a diagnostic interview that combines structured and semi-structured features. These data were reviewed by an experienced child psychiatrist (IH), and ICD10 psychiatric diagnoses were derived. Normative data using these measures have previously been obtained on a representative sample of 10438 British 5-15 year olds. ${ }^{18}$ These normative data were used for comparison. Disorders were 


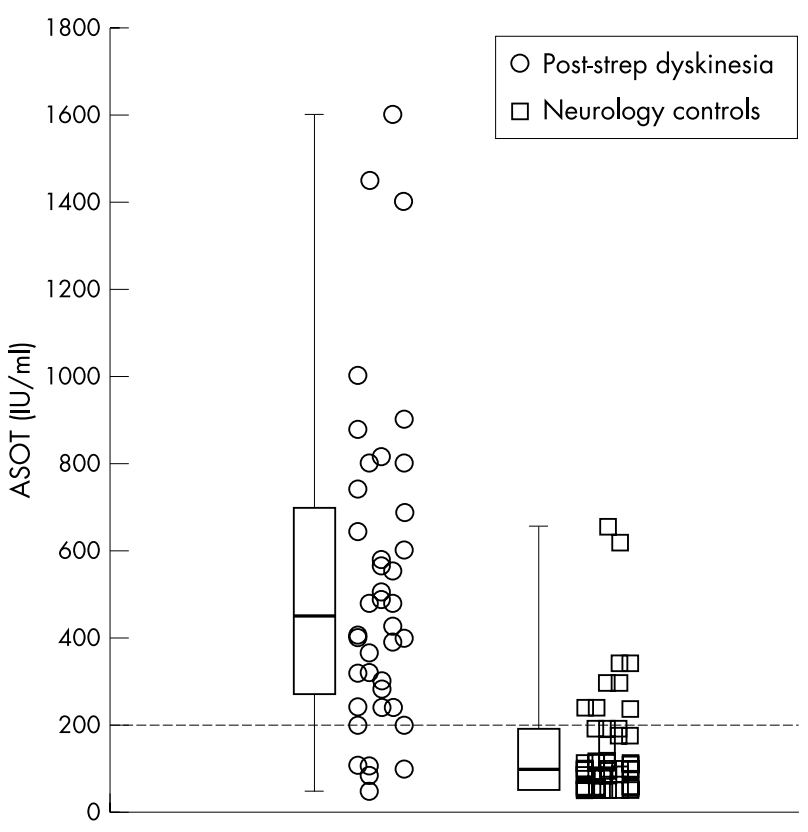

Figure 1 ASOT in post-streptococcal dyskinesia and neurological controls. Bars presented are 0th, 25th, 50th, 75th, and 100th centiles.

defined as acute if less than one year duration, chronic if more than one year.

\section{Streptococcal serology}

Anti-streptolysin $\mathrm{O}$ titres and anti-DNase $\mathrm{B}$ titres were measured using the Dade Behring BN II nephelometer. All controls were within acceptable parameters. ASOT greater than $200 \mathrm{IU} / \mathrm{ml}$ and anti-DNase B greater than $300 \mathrm{IU} / \mathrm{ml}$ were considered significant according to WHO guidelines. Serology was repeated 3-6 months later to confirm reduction in titres. In order to establish the prevalence of positive streptococcal serology in a childhood population, controls with other neurological disease of metabolic, infectious, and other aetiologies $(n=100$, mean age 7.5 years, median age 7 years, 50 males; see accompanying paper ${ }^{49}$ ), were recruited steadily over the same time period from the same institution.

\section{Statistics}

Clinical differences between patient groups and controls or normative data were examined using the $\chi^{2}$ test or the Mann-Whitney test as appropriate. Anti-streptolysin-O and anti-DNase B titres in patients and controls were analysed using the non-parametric Mann-Whitney test.

\section{RESULTS}

\section{Patients}

Table 1 provides details of the patients with respect to movement and psychiatric disorders.

\section{Age and sex}

The age range of movement disorder onset was $1.2-16$ years (mean 7.25 years, median 7 years); 21 patients were male, 19 female.

\section{Preceding medical history}

Psychiatric symptoms were present before movement disorder onset in seven patients $(17.5 \%)$ : attention deficithyperactivity disorder, $\mathrm{n}=6$ (one with co-morbid OCD and generalised anxiety); and post-traumatic stress disorder, $\mathrm{n}=1$. Other past medical history included cows' milk protein

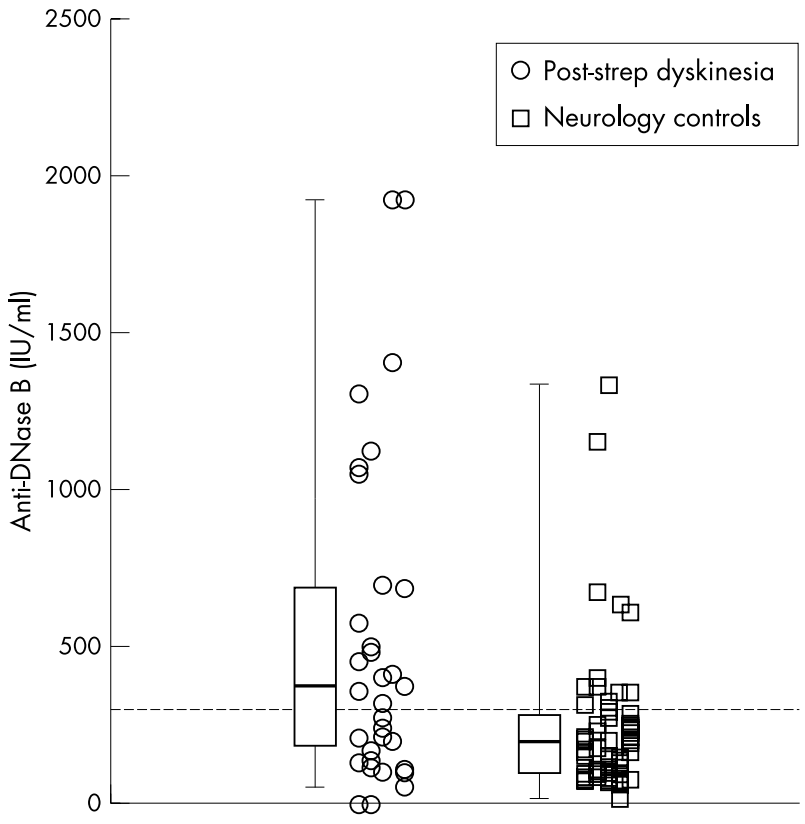

Figure 2 Anti-DNase B in post-streptococcal dyskinesia and neurological controls. Bars presented are 0 th, 25th, 50th, 75th, and 100th centiles.

allergy $(n=1)$, asthma $(n=1)$, and moya-moya disease $(\mathrm{n}=1)$.

\section{Precipitating infectious illness}

Thirty four patients (85\%) had an infectious illness compatible with BHS shortly before movement disorder onset. The illnesses were described as sore throat/tonsillitis $(n=22)$, upper respiratory tract infection $(n=9)$, cervical lymphadenopathy $(n=2)$, and scarlet fever $(n=1)$. The remaining six patients did not have a clinical history of BHS infection at movement disorder onset, but subsequently had two or more relapses associated with streptococcal infections. BHS organisms were grown from pharyngeal cultures in six patients (five group A, one group G). Streptococcal serology provided evidence of BHS infection in all other patients. The mean ASOT values were statistically increased in the dyskinesia cohort $(\mathrm{n}=40$, median $453 \mathrm{IU} / \mathrm{ml}$, mean $529 \mathrm{IU} / \mathrm{ml}$, confidence intervals of mean 415-643) compared to the neurology controls (median $100 \mathrm{IU} / \mathrm{ml}$, mean $151 \mathrm{IU} / \mathrm{ml}$, confidence intervals of mean 102-200) ( $p<0.000)$. Likewise, anti-DNase B was statistically increased in the dyskinesia cohort (median $373 \mathrm{IU} / \mathrm{ml}$, mean $566 \mathrm{IU} / \mathrm{ml}$, confidence intervals of mean 396-736) compared to the neurology control groups (median $196 \mathrm{IU} / \mathrm{ml}$, mean $213 \mathrm{IU} / \mathrm{ml}$, confidence intervals of mean 128-298 IU/ml) $(\mathrm{p}<0.000)$ (figs $\mathrm{l}$ and 2$)$. The mean latency between infection and movement disorder onset was 18.9 days (range 1-67 days).

\section{Movement disorder (table 1)}

The movement disorder onset was often abrupt, regardless of the dyskinesia phenotype. The most frequent dyskinesias were chorea $(n=20)$ and motor tics $(n=16)$. The first recorded motor tics were eye blinking/eye deviation $(n=4)$, head flicks $(n=3)$, assorted facial tics $(n=4)$, shoulder or upper limb tics $(n=3)$, and truncal tics $(n=2)$. Other dyskinesias were dystonia $(n=5)$, tremor $(n=3)$, stereotypies $(\mathrm{n}=2)$, opsoclonus $(\mathrm{n}=2)$, and myoclonus $(\mathrm{n}=1)$. One patient with dystonia, and one patient with episodic (paroxysmal) dystonic choreoathetosis, have been previously 


\begin{tabular}{|c|c|c|c|}
\hline \multirow[b]{2}{*}{ ICD-10 diagnosis } & \multicolumn{2}{|l|}{ Point prevalence } & \multirow[b]{2}{*}{ p value* } \\
\hline & Dyskinesia group & Normative data $^{18}$ & \\
\hline Any ICD-10 diagnosis & $62.5 \%$ & $8.9 \%$ & 0.000 \\
\hline Emotional disorder & $47.5 \%$ & $4.3 \%$ & 0.000 \\
\hline Obsessive-compulsive disorder & $27.5 \%$ & $0.2 \%$ & 0.000 \\
\hline Generalised anxiety & $25.0 \%$ & $0.6 \%$ & 0.000 \\
\hline Depressive episode & $17.5 \%$ & $0.7 \%$ & 0.000 \\
\hline Separation anxiety & $7.5 \%$ & $0.8 \%$ & 0.000 \\
\hline Specific phobia & $7.5 \%$ & $1.0 \%$ & 0.001 \\
\hline Social phobia & $5.0 \%$ & $0.3 \%$ & 0.000 \\
\hline Panic attacks & $2.5 \%$ & $0.1 \%$ & 0.06 \\
\hline Conduct disorders & $27.5 \%$ & $4.7 \%$ & 0.000 \\
\hline Oppositional defiant disorder & $15.0 \%$ & $2.5 \%$ & 0.000 \\
\hline Other conduct disorders & $12.5 \%$ & $2.2 \%$ & 0.000 \\
\hline Hyperkinetic disorders & $15.0 \%$ & $1.3 \%$ & 0.000 \\
\hline Hyperkinesis & $12.5 \%$ & $1.1 \%$ & 0.000 \\
\hline Other hyperkinetic disorder & $2.5 \%$ & $0.2 \%$ & 0.10 \\
\hline Less common disorders & $10 \%$ & $0.5 \%$ & 0.000 \\
\hline
\end{tabular}

described. ${ }^{6}{ }^{19}$ Seventeen patients had one or more vocal tics: 13 associated with motor tics, two with chorea, one with myoclonus, and one with stereotypies.

\section{Psychiatric disorders (tables 1 and 2)}

Acute emotional and/or behavioural alteration occurred in 33 patients $(82.5 \%)$. Most frequently reported acute changes were emotional lability $(\mathrm{n}=13,32.5 \%)$, anxiety $(\mathrm{n}=11$, $27.5 \%)$, obsessions and/or compulsions $(\mathrm{n}=9,22.5 \%)$, and depression $(\mathrm{n}=7,17.5 \%)$. Other common behavioural changes included aggressive, oppositional, or disruptive behaviours $(\mathrm{n}=14,35 \%)$ and attention deficit $(\mathrm{n}=11$, $17.5 \%)$. Less common psychiatric manifestations included echolalia $(n=4)$, visual hallucinations $(n=2)$, and social disinterest $(n=2)$. Formal psychiatric assessments were carried out at least two weeks after the acute presentation. One or more ICD-10 diagnoses were made in 25 patients $(62.5 \%)$. Thirteen patients $(32.5 \%)$ had two or more psychiatric diagnoses. Table 2 presents the ICD-10 diagnoses, with comparison to previously derived normative data. ${ }^{18}$

\section{Other neurological features}

Hypotonia and bulbar dysfunction were common in the chorea subgroup (dysarthria occurred in half of the chorea patients). Sleep disorder occurred in nine patients $(22.5 \%)$, particularly insomnia during the acute phases. Less common features included reduced consciousness $(n=2)$, epileptic seizures $(n=2)$, and mutism $(n=2)$.

\section{Systemic features}

Systemic complications occurred in nine patients $(22.5 \%)$, and affected the chorea patients only $(47.5 \%$ of chorea patients had systemic features); five had carditis defined using echocardiography (four with mitral regurgitation). Two patients had arthritis, and three other patients had arthralgia. One further patient had a vasculitic rash, but no patients had erythema marginatum. Systemic complications always preceded the neurological syndrome.

\section{Family history}

Sixteen patients $(40 \%)$ had a family history of psychiatric or movement disorders in first degree family members. Ten patients $(25 \%)$ had at least one family member with a history of movement disorders: motor tics in childhood $(n=7)$, Sydenham's chorea $(n=2)$, and Tourette syndrome $(n=1)$. Family history of psychiatric disorders (ICD-10) was present in 11 patients $(27.5 \%)$ : obsessive-compulsive disorder $(\mathrm{n}=5)$, depression $(\mathrm{n}=4)$, hyperactivity $(\mathrm{n}=3)$, anxiety, conduct disorder, and chronic fatigue syndrome $($ all $n=1)$ (some family members had more than one psychiatric diagnosis).

A family history of post-streptococcal autoimmune complications in first or second degree family members was present in eight patients $(20 \%)$ : Sydenham's chorea $(n=3)$, PANDAS $(n=3)$, and rheumatic carditis $(n=2)$.

\section{Subsequent movement disorder outcome (table 1)}

Patients with persistent disease are still under medical care. The mean duration of disease in this cohort is currently 2.7 years (2 months-13 years). Eleven (27.5\%) patients have had a monophasic disorder with complete resolution. Of the 29 patients $(72.5 \%)$ with continuing symptoms, 15 (37.5\%) have persistent static disease and $14(35 \%)$ have a relapsing remitting course associated with further infections. All of the tic patients fulfilled a diagnosis of PANDAS. ${ }^{5}$ Although there was evidence of streptococcal infections during the majority of exacerbations, two patients had relapses after apparent viral infections, and one patient had an exacerbation after a routine vaccination. Nine of the 16 patients with motor tics $(56 \%)$ fulfil a diagnosis of Tourette syndrome ICD-10.

Table 3 Clinical comparison of tic and chorea subgroups. Psychiatric diagnoses according to ICD-10 criteria

\begin{tabular}{|c|c|c|c|}
\hline Characteristic & $\begin{array}{l}\text { Tic } \\
(n=16)\end{array}$ & $\begin{array}{l}\text { Chorea } \\
(n=20)\end{array}$ & $\mathrm{p}$ value \\
\hline $\begin{array}{l}\text { Mean age of presentation } \\
\text { (range) }\end{array}$ & $\begin{array}{l}6.75 \\
(2-13)\end{array}$ & $\begin{array}{l}7.85 \\
(1.5-13)\end{array}$ & NS \\
\hline Proportion male & $69 \%$ & $35 \%$ & 0.04 \\
\hline $\begin{array}{l}\text { Mean length of follow up } \\
\text { (range) }\end{array}$ & $\begin{array}{l}3.84 \\
(0.2-13)\end{array}$ & $\begin{array}{l}1.1 \\
(0.2-17)\end{array}$ & 0.001 \\
\hline Continuing movement disorder & $94 \%$ & $55 \%$ & 0.01 \\
\hline Any psychiatric disorder & $69 \%$ & $50 \%$ & NS \\
\hline Emotional disorder & $50 \%$ & $40 \%$ & NS \\
\hline $\begin{array}{l}\text { Obsessive-compulsive } \\
\text { disorder }\end{array}$ & $50 \%$ & $10 \%$ & 0.02 \\
\hline Generalised anxiety & $19 \%$ & $25 \%$ & NS \\
\hline Major depression & $12.5 \%$ & $20 \%$ & NS \\
\hline Conduct disorders (all types) & $25 \%$ & $20 \%$ & NS \\
\hline $\begin{array}{l}\text { Hyperkinetic disorders } \\
\text { (all types) }\end{array}$ & $19 \%$ & $15 \%$ & NS \\
\hline
\end{tabular}




\section{Clinical difference between tic and chorea subgroups (table 3)}

Table 3 presents comparisons between the clinical features.

\section{DISCUSSION}

We report our experience of post-streptococcal dyskinetic movement disorders in a tertiary care setting. Historically, differentiation of chorea, tics, and myoclonus has sometimes been difficult in the context of "Sydenham's chorea", and sometimes two or more movement disorder phenotypes coexist. ${ }^{2}$ Indeed, Kerbeshian et al described a recent case of post-streptococcal tics in the context of Sydenham's chorea. ${ }^{20}$ It is perhaps not surprising that a variety of movement disorders may occur in the context of post-streptococcal neurological disease. Rarely do basal ganglia syndromes result in only one extrapyramidal phenotype; for example, although chorea is the classical phenotype of Huntington's disease, motor tics and akinetic rigid phenotypes are also recognised..$^{21} 22$

Recently, the spectrum of post-streptococcal movement disorders has been expanded beyond chorea to include motor and vocal tics, ${ }^{45}$ dystonia, ${ }^{6}{ }^{23}$ and myoclonus. ${ }^{24}$ In this report, chorea and tics were the most prevalent phenotypes, although this study was not epidemiological in design and therefore does not represent a community sample. It was possible to classify the majority of dyskinesias without difficulty, although occasionally mixed movement disorders were present. By analysing differences between the chorea and tic subgroup, we attempted to determine what variables may dictate phenotypic expression. Although the patient age did not clearly influence the dyskinesia phenotype, the sex distribution showed male predominance in the tic cohort and female predominance in the chorea subgroup. This sex distribution has been previously reported in Sydenham's chorea after puberty ${ }^{25}$ and tic disorders, ${ }^{26}$ and may suggest an influence of sex hormones on phenotypic expression. The fact that oestrogen can precipitate chorea (chorea gravidarum and oral contraceptive pill) supports the possible role of oestrogen in the chorea phenotype. ${ }^{27}$ It was notable that there was a modestly increased prevalence of neuropsychiatric disorders (particularly ADHD) preceding movement disorder onset. It is possible that ADHD could represent a specific neurodevelopmental risk factor for the later development of poststreptococcal movement disorders. Alternatively, the preceding psychiatric symptoms may be a consequence of previously unrecognised episodes of post-streptococcal autoimmunity. Only longitudinal and epidemiological studies would address these alternate hypotheses. It is also possible that the movement disorder phenotype is partly related to the particular cortico-striatal tracts involved in disease pathogenesis. ${ }^{28}$

Vocal tics were common in the motor tic subgroup; however they were not exclusive to this group. Indeed, vocal tics are an unusual but previously recognised feature of Sydenham's chorea. ${ }^{29}$ Other than extrapyramidal movements, additional neurological features were uncommon, although sleep disturbance (particularly insomnia) occurred in a significant proportion. It could be argued that the sleep disturbance is due to the disruption of normal sleep patterns by extra movements. Alternatively, the aberrant neurochemistry producing the movement disorder could also affect sleep pathways. Recent reports of sleep disturbance in Parkinson's disease and Huntington's disease support this putative hypothesis. ${ }^{30} 31$

Emotional and behavioural alteration was a common accompanying feature of the acute disease, regardless of the movement phenotype. The acute behavioural changes were often dramatic and rapid. Frequently, the children suffered a "change in personality", and became emotionally labile or aggressive. After the acute phase, formal interview showed a high prevalence of ICD-10 psychiatric diagnoses. As shown in previous cohorts of SC and PANDAS, ${ }^{59}$ emotional disorders (particularly obsessive-compulsive disorder and anxiety disorders) were the most common, and the incidence of obsessive-compulsive disorder (OCD) was more common in chronic or relapsing SC. ${ }^{32}$ Indeed, OCD in this cohort was limited to the patients whose movement disorder had been present for more than one year. The shorter mean duration of illness in the SC cohort may be responsible for the reduced incidence of OCD in this subgroup. Otherwise, there were no clear differences in the psychiatric morbidity between the chorea and tic subgroups in this study. In addition to emotional disorders, conduct disorders and hyperkinetic disorders (such as ADHD) were also common. By contrast, psychotic symptoms were rare, although they have been occasionally described in Sydenham's chorea. ${ }^{33}$ It is important to note that the psychiatric complications frequently remain long after the acute movement disorder has resolved. ${ }^{7}$

The main difference between the chorea and tic subgroups was the presence of systemic features (carditis and arthritis) that were exclusive to the chorea subgroup. The cause of this important difference is unknown although we acknowledge that systematic cardiac examination in PANDAS patients has not been performed in this, or other case series.

Although some patients within this cohort have had transient disease or resolution of the movement disorder within a year, a large proportion of patients have had persistent or relapsing disease. The high proportion of patients with protracted disease may be due to selection bias, as patients with milder transient disease are unlikely to be referred to a tertiary referral centre. Previous studies suggest that Sydenham's chorea becomes persistent in 20$50 \%$ of patients. ${ }^{25}{ }^{34}$ Swedo's proposed diagnostic criteria for PANDAS require two or more exacerbations of motor tics or OCD with streptococcal infections. ${ }^{5}$ All of the tic patients in this cohort fulfil a diagnosis of PANDAS. If we believe that Sydenham's chorea and PANDAS are biologically similar disorders, Swedo's clinical criteria would exclude a significant proportion of patients with monophasic disorders. Laboratory confirmation of recent BHS infection is necessary for a diagnosis of post-streptococcal dyskinesia; however positive BHS serology is prevalent in paediatric populations and is unlikely to be a specific diagnostic tool used in isolation. Indeed, $18 \%$ of the neurological controls had increased streptococcal serology. Previous childhood control groups in the USA have even higher rates of positive streptococcal serology. ${ }^{35}$ This has consequently lead to diagnostic difficulty, and even questioned whether the "PANDAS" phenotype exists (critically reviewed by Singer and Loiselle $\left.{ }^{36}\right)$. The proposed mediators of disease are antibodies reactive against brain epitopes. Anti-brain antibodies appear to be a useful marker in acute Sydenham's chorea and post-streptococcal dystonia, ${ }^{637}$ although their usefulness in PANDAS (and Tourette syndrome) is more contentious. ${ }^{38}$ Ninety three per cent of the dyskinetic patients reported in this paper had positive anti-brain antibodies using these methods (paper attached).

The prevalence of movement and emotional disorders in first degree family relatives suggests that a genetic predisposition is important in disease development, in addition to the environmental trigger. ${ }^{39}$ It could be argued that the high incidence of neuropsychiatric disease in first degree family members is due to a genetically determined neurodevelopmental vulnerability. Alternatively, the psychiatric disease in parents and siblings could have had a psychological impact on the patients. Our more favoured hypothesis is that disease is related to a genetically determined autoimmune predisposition. The positive family history of post-streptococcal 
autoimmune syndromes (Sydenham's chorea, PANDAS, and rheumatic fever) in this study and previous cohorts of SC supports this genetic immune hypothesis. ${ }^{25}$ It has been recognised that patients with rheumatic fever have a high expression of a B lymphocyte surface marker, D8/17.40 Patients with Sydenham's chorea and PANDAS also have significantly higher expression of this lymphocyte marker compared to healthy and autoimmune controls, although the importance of this marker is unknown. ${ }^{40-42}$ Finally, it is also possible that genetic vulnerability is mediated via a combination of these mechanisms (neurochemical, immunological, and psychological).

This report does not address the important issue of treatment. Although penicillin prophylaxis throughout childhood has been shown to be effective at reducing relapses in rheumatic fever and $\mathrm{SC}^{43}$ no similar study has been adequately performed in PANDAS. ${ }^{44}$ However, one group reported that prompt antibiotic treatment of pharyngeal infections lead to an improvement in PANDAS neuropsychiatric symptoms. ${ }^{45}$ Although immune therapies such as steroids, immunoglobulin, and plasma exchange have been successfully used in SC and PANDAS, the significant side effects of these therapies precludes routine use until further studies prove their benefit. ${ }^{46}{ }^{47}$

In conclusion, a broad spectrum of extrapyramidal movements and neuropsychiatric disorders may occur after BHS infection. Although the clinical similarities between the chorea and tic subgroups suggest that SC and PANDAS may be two phenotypes of the same immune mediated basal ganglia disorder, other authors have highlighted the differences. ${ }^{48}$ Phenotypic expression may depend on other variables including the specific cortico-striatal circuits involved, developmental status, genetic predisposition, and patient's sex. There is phenotypic similarity between post-streptococcal CNS syndromes and common neuropsychiatric disease such as tic disorders, Tourette syndrome, ADHD, and OCD. Improved understanding of the disease mechanism in poststreptococcal CNS disease could significantly improve our knowledge of the neurochemistry and neuroanatomy of common childhood diseases.

\section{ACKNOWLEDGEMENTS}

This work was undertaken by Great Ormond Street Hospital for Children NHS Trust who received a proportion of its funding from the NHS Executive; the views expressed in this publication are those of the authors and not necessarily those of the NHS Executive. RCD has a research training fellowship awarded by Action Research, and the Barnwood House trust, UK.

\section{Authors' affiliations}

R C Dale, I Heyman, R A H Surtees, B G R Neville, Neurosciences Unit, Institute of Child Health and Great Ormond Street Hospital for Children NHS Trust, London, UK

A J Church, G Giovannoni, Department of Neuroinflammation, Institute of Neurology, London, UK

R Goodman, Child and Adolescent Psychiatry Unit, Institute of Psychiatry, London, UK

\section{REFERENCES}

1 Fernandez-Alvarez E, Aicardi J. General concepts. In: Fernandez-Alvarez E, Aicardi J, eds. Movement disorders in children. London: MacKeith Press, 2001:1-24.

2 Creak M, Guttman E. Chorea, tics, compulsive utterances. J Med Sci 1935;81:834.

3 Osler WM. Quoted in: Sacks O. "Acquired Tourettism in adult life" in Gilles de la Tourette syndrome. In: Friedhoff AJ, Chase TN, eds. Advances in neurology. New York: Raven Press, 1982;35:89-92.

4 Kiessling LS, Marcotte AC, Culpepper L. Antineuronal antibodies in movement disorders. Pediatrics 1993;92:39-43.

5 Swedo SE, Leonard HL, Garvey M, et al. Pediatric autoimmune neuropsychiatric disorders associated with streptococcal infections: clinical description of the first 50 cases. Am J Psychiatry 1998;155:264-71.
6 Dale RC, Church AJ, Benton S, et al. Post-streptococcal autoimmune dystonia with isolated bilateral striatal necrosis. Dev Med Child Neurol 2002;44:485-9.

7 Freeman JM, Aron AM, Collard JE, et al. The emotional correlated of Sydenham's chorea. Pediatrics 1965;35:42-9.

8 Swedo SE, Rapoport JL, Cheslow DL, et al. High prevalence of obsessivecompulsive symptoms in patients with Sydenham's chorea. Am J Psychiatry 1989;146:246-9.

9 Mercadante MT, Busatto GF, Lombroso PJ, et al. The psychiatric symptoms of rheumatic fever. Am J Psychiatry 2000;157:2036-8.

10 Marie P, Tretiakoff C. Examen histologique des centres nerveux dans un cas de choree aigue de Sydenham. Rev Neurol 1920;36:428-38.

11 Greenfield JG, Wolfsohn JM. The pathology of Sydenham's chorea. Lancet 1922;2:603-6.

12 Giedd JN, Rapoport JL, Kruesi MJ, et al. Sydenham's chorea: magnetic resonance imaging of the basal ganglia. Neurology 1995;45:2199-202.

13 Giedd JN, Rapoport JL, Garvey MA, et al. MRI assessment of children with obsessive-compulsive disorder or tics associated with streptococcal infection. Am J Psychiatry 2000;157:281-3.

14 Husby G, van de Rijn I, Zabriskie JB, et al. Antibodies reacting with cytoplasm of subthalamic and caudate nuclei neurons in chorea and acute rheumatic fever. J Exp Med 1976;144:1094-110.

15 Jankovic J. International Classification of Diseases, tenth revision: neurological adaptation (ICD-10 NA): extrapyramidal and movement disorders. Mov Disord 1995; 10:533-40.

16 Goodman R. Psychometric properties of the strengths and difficulties questionnaire. J Am Acad Child Adolesc Psychiatry 2001;40:1337-45.

17 Goodman R, Ford T, Richards H, et al. The Development and Well-Being Assessment: description and initial validation of an integrated assessment of child and adolescent psychopathology. J Child Psychol Psychiatry 2000;41:645-55.

18 Meltzer H, Gatward R, Goodman R, et al. The mental health of children and adolescents in Great Britain. London: HMSO, 2000.

19 Dale RC, Church AJ, Surtees RAH, et al. Post-streptococcal autoimmune CNS disease presenting as paroxysmal dystonic choreoathetosis. Mov Disord 2002; 17:817-20

20 Kerbeshian J, Burd L, Pettit R. A possible post-streptococcal movement disorder with chorea and tics. Dev Med Child Neurol 1990;32:642-4

21 van Dijk JG, van der Velde EA, Roos RA, et al. Juvenile Huntington disease. Hum Genet 1986;73:235-9.

22 Jankovic J, Ashizawa T. Tourettism associated with Huntington's disease. Mov Disord 1995; 10:103-5.

23 Dale RC, Church AJ, Cardoso F, et al. Poststreptococcal acute disseminated encephalomyelitis with basal ganglia involvement and auto-reactive antibasal ganglia antibodies. Ann Neurol 2001;50:588-95.

24 Difazio MP, Morales J, Davis R. Acute myoclonus secondary to group A betahemolytic streptococcus infection: a PANDAS variant. J Child Neurol 1998;13:516-18.

25 Nausieda PA, Grossman BJ, Koller WC, et al. Sydenham chorea: an update. Neurology 1980;30:331-4.

26 Singer HS, Walkup JT. Tourette syndrome and other tic disorders. Diagnosis, pathophysiology, and treatment. Medicine (Baltimore) 1991;70:15-32.

27 Nausieda PA, Koller WC, Weiner WJ, et al. Chorea induced by oral contraceptives. Neurology 1979;29:1605-9.

28 Albin RL, Young AB, Penney JB. The functional anatomy of basal ganglia disorders. Trends Neurosci 1989;12:366-75.

29 Mercadante MT, Campos MC, Marques-Dias MJ, et al. Vocal tics in Sydenham's chorea. J Am Acad Child Adolesc Psychiatry 1997;36:305-6.

30 Silvestri R, Raffaele M, De Domenico $P$, et al. Sleep features in Tourette's syndrome, neuroacanthocytosis and Huntington's chorea. Neurophysiol Clin 1995;25:66-77.

31 Rye DB, Jankovic J. Emerging views of dopamine in modulating sleep/wake state from an unlikely source: PD. Neurology 2002;58:341-6.

32 Asbahr FR, Ramos RT, Negrao AB, et al. Case series: increased vulnerability to obsessive-compulsive symptoms with repeated episodes of Sydenham chorea. J Am Acad Child Adolesc Psychiatry 1999;38:1522-5.

33 Moore DP. Neuropsychiatric aspects of Sydenham's chorea: a comprehensive review. J Clin Psychiatry 1996;57:407-14.

34 Cardoso F, Vargas AP, Oliveira LD, et al. Persistent Sydenham's chorea. Mov Disord 1999; 14:805-7.

35 Singer HS, Giuliano JD, Hansen BH, et al. Antibodies against human putamen in children with Tourette syndrome. Neurology 1998;50:1618-24

36 Singer HS, Loiselle C. PANDAS. A commentary. J Psychosom Res 2003:55:31-9.

37 Church AJ, Cardoso F, Dale RC, et al. Anti-basal ganglia antibodies in acute and persistent Sydenham's chorea. Neurology 2002;59:227-31.

38 Loiselle CR, Wendlandt JT, Rohde CA, et al. Antistreptococcal, neuronal, and nuclear antibodies in Tourette syndrome. Pediatr Neurol 2003;28:119-25.

39 Lougee L, Perlmutter SJ, Nicolson R, et al. Psychiatric disorders in first-degree relatives of children with pediatric autoimmune neuropsychiatric disorders associated with streptococcal infections (PANDAS). J Am Acad Child Adolesc Psychiatry 2000;39:1120-6.

40 Khanna AK, Buskirk DR, Williams RC Jr, et al. Presence of a non-HLA B cell antigen in rheumatic fever patients and their families as defined by a monoclonal antibody. J Clin Invest 1989;83:1710-16.

41 Gibofsky A, Khanna A, Suh E, et al. The genetics of rheumatic fever: relationship to streptococcal infection and autoimmune disease. J Rheumatol 1991;30(suppl): 1-5.

42 Swedo SE, Leonard $\mathrm{HL}$, Mittleman BB, et al. Identification of children with pediatric autoimmune neuropsychiatric disorders associated with 
streptococcal infections by a marker associated with rheumatic fever. Am J Psychiatry 1997; 154:110-12.

43 Dajani AS, Bisno AL, Chung KJ, et al. Prevention of rheumatic fever: a statement for health professionals by the Committee on Rheumatic Fever, Endocarditis and Kawasaki Disease of the Council on Cardiovascular Disease in the young, the American Heart Association. Pediatr Infect Dis J 1989;8:263-6.

44 Garvey MA, Perlmutter SJ, Allen AJ, et al. A pilot study of penicillin prophylaxis for neuropsychiatric exacerbations triggered by streptococcal infections. Biol Psychiatry 1999;45:1564-71.

45 Murphy ML, Pichichero ME. Prospective identification and treatment of children with pediatric autoimmune neuropsychiatric disorder associated with group A streptococcal infection (PANDAS). Arch Pediatr Adolesc Med 2002; 156:356-61.

46 Green LN. Corticosteroids in the treatment of Sydenham's chorea. Arch Neurol 1978;35:53-4.

47 Perlmutter SJ, Leitman SF, Garvey MA, et al. Therapeutic plasma exchange and intravenous immunoglobulin for obsessive-compulsive disorder and tic disorders in childhood. Lancet 1999;354:1153-8.

48 Murphy TK, Goodman WK, Ayoub EM, et al. On defining Sydenham's chorea: where do we draw the line? Biol Psychiatry 2000;47:851-7.

49 Church AJ, Dale RC, Giovannoni G. Anti-basal ganglia antibodies: a possible diagnostic utility in idiopathic movement disorders? Arch Dis Child 2004;89:611-4.

\section{ARCHIVIST}

\section{Randomisation understood but not accepted}

T

here is evidence that patients may neither understand nor accept randomisation in clinical trials. In a study in North Staffordshire (Cicely Kerr and colleagues. Journal of Medical Ethics 2004;30:80-4) adults attending further education classes appeared to understand randomisation but not accept it.

The 130 participants in the study were aged between 18 and 70 (mean 32 years), 67\% were women, and their occupations ranged from unskilled to managerial or professional. Most were not formally educated beyond GCSE or O levels (usually taken at 16 years). They were given two hypothetical scenarios (one medical and one nonmedical) in which people needed to be allocated to two groups. Five methods of allocation were proposed: computer allocation with no information about individuals, toss of a coin, drawing from a hat, personal preference, or allocation in turn. They were asked to decide for each of these methods whether it was random or not random. Most participants (75-90\%) judged that computer allocation, toss of a coin, and drawing from a hat were random methods and asking people for their preference was not random. They were more or less equally divided about the status of allocation-in-turn. Similar answers were given whether considering the medical or the nonmedical scenario.

Participants were then asked to imagine they were to take part in a clinical trial comparing two drugs both known to be beneficial. They had to decide which of the same five methods would be acceptable for allocation to one or the other drug. Half were given a brief written justification for the use of randomisation and half were not. Among participants who correctly judged each method to be random or not random in the first part of the study most (60\% if given the written justification for randomisation, $75 \%$ if not) considered allocation by patient preference acceptable. Most (62-72\% of the group not given the written justification) thought that computer allocation, toss of a coin, drawing from a hat, or allocation-in-turn were not acceptable. The written justification did not change attitudes except towards computer randomisation which was acceptable to $38 \%$ of participants not given the justification and 58\% of those given it.

Most people are able to distinguish between random and non-random methods of allocation but most would not find randomisation acceptable in a clinical trial. Computer allocation is seen as more appropriate than tossing a coin or drawing from a hat. More needs to be done to explain the reasons for randomisation in more detail and to address people's questions.

Question-If some people choose drug A and some drug B, what's wrong with giving them their choice and seeing how they get on? Answer-To obtain reliable results we must compare like with like. The purpose of randomisation is not just to allocate people to groups but to try to make sure that the groups are as alike as possible; one group is not older, sicker, or different from the other in any way that might affect their response to treatment. With large enough numbers of people any differences should be evened out by randomisation; chance should make it likely that more or less the same numbers of older or sicker people (or those different in ways nobody has thought of) are allocated to each group. Giving people their choice would not achieve that and the results of the trial would be unreliable; we would still not know for sure which drug was better. It would, of course, be completely unethical to allocate anybody to a treatment known beforehand to be inferior. ResponseO.K. I'll buy that. 